\title{
Facilitation of Affiliation and Pair-Bond Formation by Vasopressin Receptor Gene Transfer into the Ventral Forebrain of a Monogamous Vole
}

\author{
Lauren J. Pitkow, ${ }^{1}$ Catherine A. Sharer, ${ }^{1}$ Xianglin Ren, ${ }^{2}$ Thomas R. Insel, ${ }^{1}$ Ernest F. Terwilliger, ${ }^{2}$ and \\ Larry J. Young ${ }^{1}$ \\ ${ }^{1}$ Center for Behavioral Neuroscience and Department of Psychiatry, Emory University, Atlanta, Georgia 30322, and \\ 2Harvard Institutes of Medicine and Beth Israel Deaconess Medical Center, Boston, Massachusetts 02215
}

Behaviors associated with monogamy, including pair-bond formation, are facilitated by the neuropeptide vasopressin and are prevented by a vasopressin receptor [V1a receptor (V1aR)] antagonist in the male prairie vole. The neuroanatomical distribution of $\mathrm{V} 1 \mathrm{aR}$ dramatically differs between monogamous and nonmonogamous species. V1aR binding is denser in the ventral pallidal region of several unrelated monogamous species compared with nonmonogamous species. Because the ventral pallidum is involved in reinforcement and addiction, we hypothesize that $\mathrm{V} 1 \mathrm{aR}$ activation in this region promotes pair-bond formation via a mechanism similar to conditioning. Using an adeno-associated viral vector to deliver the $V 1 a R$ gene, we increased the density of $\mathrm{V} 1 \mathrm{aR}$ binding in the ventral pallial region of male prairie voles. These males exhibited increased levels of both anxiety and affiliative behavior compared with control males. In addition, males overexpressing the V1aR in the ventral pallidal region, but not control males, formed strong partner preferences after an overnight cohabitation, without mating, with a female. These data demonstrate a role for ventral pallidal $\mathrm{V} 1 \mathrm{aR}$ in affiliation and social attachment and provide a potential molecular mechanism for species differences in social organization.

Key words: vasopressin; V1a receptor; ventral pallidum; social attachment; affiliation; viral vector; monogamy; pair bond
Affiliative behavior and social bonding are essential components of human society, yet little is known of the neural circuitry regulating these complex behavioral processes. Disruptions in these processes may be associated with psychiatric diseases characterized by social deficits, such as autism. Voles provide a useful animal model for investigating the neural mechanisms underlying these behaviors (Carter, 1998; Young et al., 1998; Insel and Young, 2001). Vole species display a wide range of social behavior, ranging from being highly social and monogamous to being solitary and promiscuous. Both field and laboratory studies have demonstrated that prairie voles (Microtus ochrogaster) are a highly gregarious species that forms enduring, selective social bonds between mates (Shapiro and Dewsbury, 1990; Carter and Getz, 1993).

Pharmacological studies in prairie vole males have demonstrated that the neuropeptide arginine vasopressin (AVP) increases affiliative behavior and is critical for the formation of the pair bond (Winslow et al., 1993; Cho et al., 1999; Young et al., 1999a). In the laboratory, the formation of the pair bond is assessed using a partner-preference paradigm in which the subject chooses to associate with either the partner or a novel female of similar stimulus value. An AVP antagonist selective for the V1a receptor (V1aR) subtype blocks the development of the partner preference when given centrally before mating (Winslow et al., 1993; Cho et al., 1999). Conversely, central infusions of AVP

\footnotetext{
Received May 16, 2001; revised July 6, 2001; accepted July 6, 2001.

This work was supported by National Institute of Mental Health Grants 58824 and 56897 (L.J.Y.) and by National Science Foundation Grant IBN 9876754 (T.R.I.).

Correspondence should be addressed to Larry J. Young, Center for Behavioral Neuroscience, 954 Gatewood Drive, Emory University, Atlanta, GA 30322. E-mail: lyoun03@emory.edu.

Copyright (C) 2001 Society for Neuroscience $\quad 0270-6474 / 01 / 217392-05 \$ 15.00 / 0$
}

facilitate partner-preference formation even in the absence of mating (Winslow et al., 1993). AVP infusions do not alter affiliative behavior in the nonmonogamous montane vole (Young et al., 1999a). These data suggest that AVP released during social interactions and during mating bouts activates neural circuitry that regulates these behaviors in a species-specific manner.

The neuroanatomical distribution of the V1aR differs dramatically among closely related species that differ in their social structure, providing a potential explanation for the speciesspecific effects of AVP (Insel et al., 1994). The monogamous prairie (Microtus ochrogaster) and pine (M. pinetorum) voles, California mouse (Peromyscus californicus), and common marmoset (Callithrix jacchus) each have high densities of V1aR binding in a region of the ventral forebrain containing the ventral pallidum and substantia innominata. In this same region, relatively little $\mathrm{V} 1 \mathrm{aR}$ binding is detectable in related nonmonogamous montane (M. montanus) and meadow (M. pennsylvanicus) voles, white-footed mice (Peromyscus leucopus), and rhesus monkeys (Insel et al., 1994; Bester-Meredith et al., 1999; Young, 1999; Young et al., 1999b).

Because the ventral pallidum has been associated with reward and conditioned place preference (McBride et al., 1999), we hypothesized that $\mathrm{V} 1 \mathrm{aR}$ in this area may facilitate affiliation and partner-preference formation through its reinforcing actions in the ventral pallidum. If this is correct, animals with high densities of $\mathrm{V} 1 \mathrm{aR}$ in this region would exhibit higher levels of affiliative behavior and be more likely to form social attachments. To test this hypothesis, we used an adeno-associated viral (AAV) vector to selectively increase V1aR expression in the ventral pallidal area of male prairie voles and examined the behavioral consequences, including anxiety, affiliation, and partner-preference formation. 


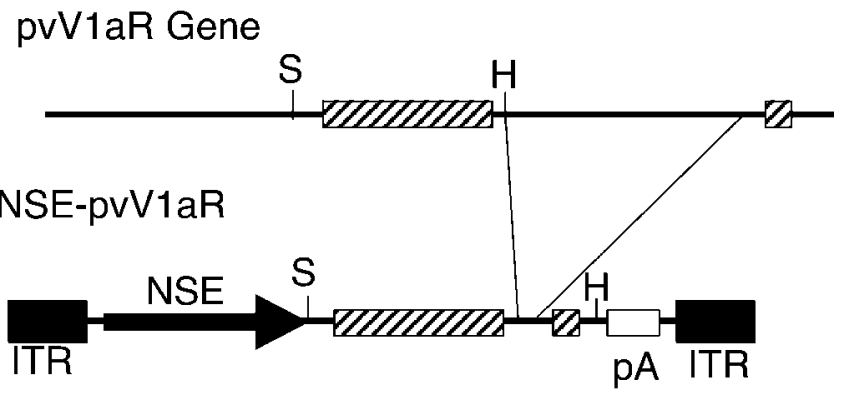

Figure 1. The NSE-pvVIaR plasmid was constructed by inserting a modified genomic prairie vole VlaR clone between the NSE promoter (arrow) and the SV40 small $\mathrm{t}$ intron and poly(A) signal ( $p A$ ) (open box). The $V 1 a R$ sequence used in the plasmid spanned from +20 relative to the transcription start site to the stop codon, with all but $287 \mathrm{bp}$ of the intron deleted. The striped box indicates VlaR exons. S, SalI; H, HindIII; ITR, viral iterated terminal repeats.

\section{MATERIALS AND METHODS}

\section{Animals and treatment}

Subjects were 2- to 5-month-old sexually naive male prairie voles $(M$. ochrogaster) reared in our laboratory from field-caught specimens. Animals were housed with one to two same-sex litter mates in a 14/10 hr light/dark cycle and were provided food (Purina Rabbit Chow; Purina Mills, St. Louis, MO) and water ad libitum. Littermates housed together were assigned to different treatment groups to control for variability within litters and within cages. All experiments were performed in compliance with the rules and oversight of the Emory Institutional Animal Care and Use Committee.

Three groups of male prairie voles were used in this study. The experimental group [V1aR-ventral pallidum (VP); $n=13$ ] received bilateral infusions of the neuron-specific enolase (NSE)-prairie vole VIaR ( $v v V 1 a R)$ viral vector into the ventral pallidal area. One control group [VlaR-caudate putamen $(\mathrm{CP}) ; n=9]$ received identical injections into the caudate putamen, a region that does not express the endogenous $\mathrm{V} 1 \mathrm{aR}$. A second control group (lacZ-VP/CP, $n=8$ ) received a control vector expressing the $l a c Z$ gene rather the $V 1 a R$, into either the ventral pallidum or the caudate putamen.

AAV infusions were performed under ketamine anesthesia in a stereotaxic apparatus fitted with an Ultra Micro Pump II (World Precision Instruments, Sarasota, FL) apparatus with a Hamilton syringe adapter. The injection coordinates were as follows (in $\mathrm{mm}$ ): ventral pallidum, 1.3 anterior, 1.0 lateral, and 5.5 ventral; caudate putamen, 1.3 anterior, 2.0 lateral, and 4.0 ventral. Bregma was used as the anterior-posterior and medial-lateral reference point, and the top of the skull at the anteriorlateral coordinate site was used as the dorsal-ventral reference point. Once the Hamilton syringe was lowered to the injection site, the recombinant AAV (rAAV) preparation $\left(1 \mu \mathrm{l} /\right.$ side at $10^{8}$ infectious units $\left./ \mu \mathrm{l}\right)$ was infused at a rate of 3-5 $\mathrm{nl} / \mathrm{sec}$. The needle was left in place for $2 \mathrm{~min}$ after the injection. All injections were bilateral. Animals recovered for $12 \mathrm{~d}$ before behavioral testing.

After the behavioral studies, brains were collected and analyzed for V1aR expression and accuracy of injection.

\section{AAV production}

The prairie vole VlaR viral vector (NSE- $p v V 1 a R$ ) consisted of a modified VlaR genomic clone with the majority of the intron removed, spliced downstream of a neuron-specific enolase promoter (Fig. 1). The VlaR cassette was created by first excising the sequence downstream of the HindIII site located 123 bp $3^{\prime}$ of the exon-intron boundary. The HindIII end was destroyed and the plasmid was recircularized to facilitate subsequent cloning steps. The second exon was amplified by PCR using a $5^{\prime}$ primer located $133 \mathrm{bp}$ upstream of the second exon and a $3^{\prime}$ primer that contained the stop codon and a HindIII restriction site. The amplified fragment was spliced downstream of the destroyed HindIII site. At the 5' end of the clone, a SalI site was inserted into the SacI site located $20 \mathrm{bp}$ downstream of the putative transcription initiation site and $227 \mathrm{bp}$ upstream of the translation initiation codon. The resulting V1aR sequence, containing a 227 bp $5^{\prime}$ untranslated region and the first and second exons separated by a 287 bp intron, was cloned into the SalI and
HindIII sites of an AAV plasmid derived from pSSV9, a genomic clone of AAV-2 (originally provided by R. J. Samulski, University of North Carolina, Chapel Hill, NC). The AAV coding sequences of pSSV9 were first excised and replaced with a $0.44 \mathrm{~kb}$ simian virus 40 (SV40) DNA fragment (SVpA) containing the SV40 small $t$ intron and poly(A) signal as well as several unique cloning sites. The viral iterated terminal repeats required for packaging remained intact in this plasmid. A PCR-amplified NSE promoter fragment (1 kb in length) and the $p v V 1 a R$ sequence were then inserted $5^{\prime}$ to the SVpA segment. Transduction by AAV-2 vectors into the brain is primarily confined to neurons (Bartlett et al., 1998), but the NSE promoter further restricts expression to neurons (Peel et al., 1997). The lac $Z$ vector was prepared in similar manner, incorporating a PCR-amplified $0.6 \mathrm{~kb}$ cytomegalovirus-immediate early promoter and the $3.7 \mathrm{~kb}$ Escherichia coli lacZ gene sequence. Packaging of all recombinant AAV plasmids was done according to standard protocols described previously, with some modifications, by co-complementation with the AAV trans-acting factors provided on a separate plasmid and an adenovirus helper (Wu et al., 1998). AAV vector stocks were titrated by real-time PCR using an Applied Biosystems Prism 7700 Sequence Detection System from Perkin-Elmer Applied Biosystems (Foster City, $\mathrm{CA})$. Titers of AAV vector preparations as determined by this technique average $10^{11} / \mathrm{ml}$.

\section{Behavior testing}

Elevated plus maze. The elevated plus maze testing was performed in an isolated behavior room at $23-24^{\circ} \mathrm{C}$. Animals were brought to the testing room $30 \mathrm{~min}$ before testing began to acclimate to the environment. The plus maze apparatus consisted of an open plank intersecting a walled plank, each measuring $1.4 \mathrm{~m}$ in length and elevated $1 \mathrm{~m}$ above the ground. The subject was placed in the center of the apparatus and its location was recorded for $10 \mathrm{~min}$.

Affiliation test. The affiliation test was conducted in a novel cage between 3:00 and 5:00 P.M. in a quiet room with a temperature of $23-24^{\circ} \mathrm{C}$. After a $30 \mathrm{~min}$ acclimation, a $29-$ to 31 -d-old juvenile male prairie vole was placed in the cage and behavior was recorded for $10 \mathrm{~min}$. Each juvenile was used for no more than two tests per day.

Partner-preference test. Partner-preference tests were performed immediately after a $17 \mathrm{hr}$ cohabitation with a nonestrous adult female. The pair were allowed to interact freely but mating did not occur during the cohabitation period because the females were not in behavioral estrous. Under these conditions, male prairie voles typically do not develop a partner preference in $<24 \mathrm{hr}$ of cohabitation unless mating occurs (Insel and Hulihan, 1995; Insel et al., 1995). The experimental male was placed in the center, neutral chamber of a three-chambered testing arena in which the partner was tethered in one chamber and a novel (stranger) female was tethered in a second chamber. The experimental animal was free to move throughout these chambers via Plexiglas connecting tubes. During the $3 \mathrm{hr}$ test, the location and proximity of the male to each female was recorded. Partner preference was defined as spending more than twice as much time in the partner's cage relative to the stranger's cage.

\section{Receptor binding analysis}

V1aR binding was localized by receptor autoradiography using a ${ }^{125} \mathrm{I}$-linear AVP antagonist (NEX 310; DuPont NEN, Boston, MA) as described previously (Young et al., 1997). Receptor density on autoradiographic film was quantified using NIH Image software on a Macintosh computer. Bilateral measurements from three sections per animal were taken, and a mean density measurement was obtained by subtracting background. Optical densities were converted to $\mathrm{dpm} / \mathrm{mg}$ tissue equivalents using ${ }^{125} \mathrm{I}$ microscales (Amersham Pharmacia Biotech, Arlington Heights, IL) as standards.

\section{RESULTS}

The animals receiving the NSE-pvVlaR vector into the ventral pallidal region $(V 1 a R-V P)$ had nearly a $100 \%$ increase in receptor density in the ventral pallidal area compared with the control groups (Fig. 2) [ANOVA, $F_{(2,24)}=6.99, p<0.004$; Fisher's least significant difference (LSD) post hoc test, $p<0.003$ and $p<0.01$ compared with V1aR-CP and lacZ-VP/CP, respectively]. Those animals receiving similar injections into the caudate putamen (V1aR-CP) also had significantly more $\mathrm{V} 1 \mathrm{aR}$ binding in the caudate relative to the $\mathrm{V} 1 \mathrm{aR}-\mathrm{VP}$ and the $l a c Z-\mathrm{VP} / \mathrm{CP}$ groups 


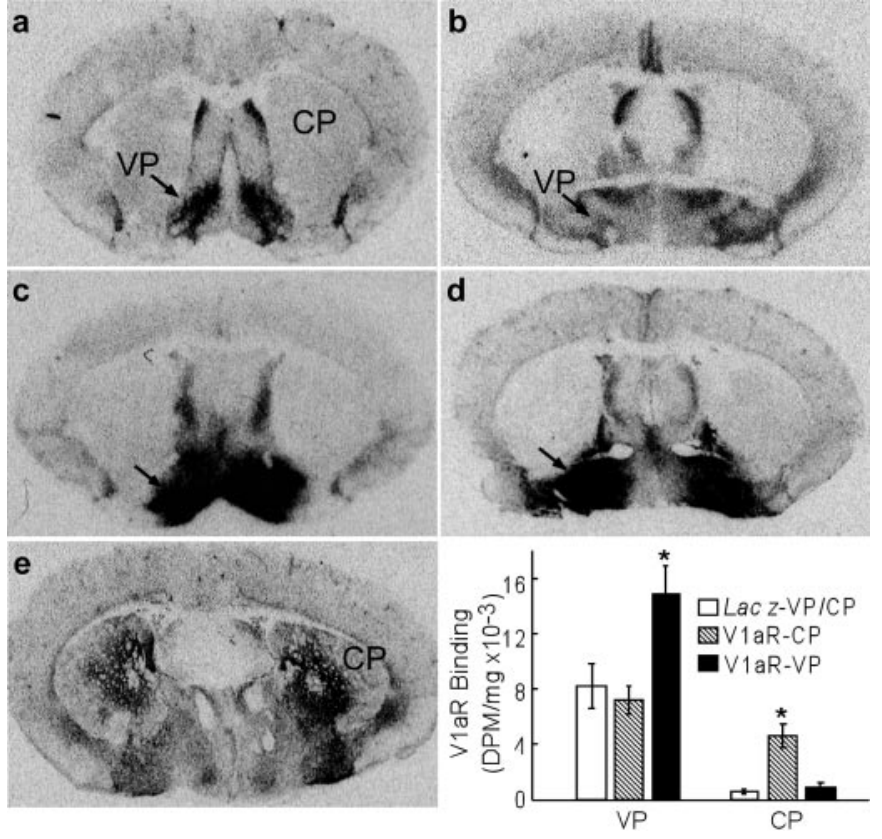

Figure 2. V1aR binding autoradiograms illustrating ${ }^{125} \mathrm{I}$-linear AVP antagonist binding in lacZ-VP/CP males $(a, b)$ and V1aR-VP males $(c, d)$ at the level of the VP (arrows). Sections $a$ and $c$ are slightly rostral to those of $b$ and $d$. The V1aR binding field in control animals is most intense in the more rostral section of the ventral pallidum. V1aR binding in the caudate putamen of the VIaR-CP males is illustrated in $e$. The quantitative analysis (mean \pm SEM) of the V1aR binding in the ventral pallidum and caudate putamen of each group is presented in the bottom right panel. ${ }^{*} p<0.01$.

(Fig. 2) (ANOVA, $F_{(2,24)}=22.6, p<0.001$; Fisher's LSD post hoc test, $p<0.001$ compared with each of the other groups). V1aR binding was not limited exclusively to the ventral pallidal nucleus in all animals because there was some spread of the virus beyond the boundaries of this nucleus; however, the ventral pallidum showed a consistent increase in $\mathrm{V} 1 \mathrm{aR}$ binding in all of the VlaR-VP animals.

Because AVP has been shown to increase anxiety in rats via a V1aR-dependent mechanism (Landgraf et al., 1995; Liebsch et al., 1996), the infected voles were first tested on an elevated plus maze to measure general anxiety. The VlaR-VP group spent significantly less time on the open arms of the plus maze compared with the control groups (Fig. $3 a$ ) (ANOVA, $F_{(2,23)}=11.43, p<0.0001$; Fisher's LSD post hoc test, $p<0.001$ compared with each control group). This suggests that increasing V1aR in the ventral pallidum results in increased anxiety in this testing paradigm.

The VlaR-VP group also displayed elevated levels of affiliative behavior relative to the control groups. Specifically, when placed in a novel cage and then presented with a novel juvenile male for a 10 min exposure, V1aR-VP animals spent significantly more time investigating and huddling (side-by-side contact) with the juvenile compared with the control groups (Fig. 3b) (ANOVA, $F_{(2,27)}=$ $4.85, p<0.004$; Fisher's LSD post hoc test, $p<0.007$ and $p<0.04$ compared with pvV1a-CP and lacZ-VP/CP, respectively). Within the VlaR-VP group, there was no significant correlation between the time spent in the open arms of the elevated plus maze and affiliative behavior.

To determine whether overexpression of V1aR altered pairbond formation, we tested for the development of a partner preference, measured as time spent with the female partner verses a a

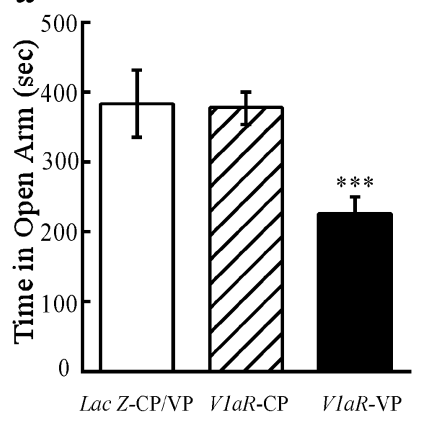

C

(

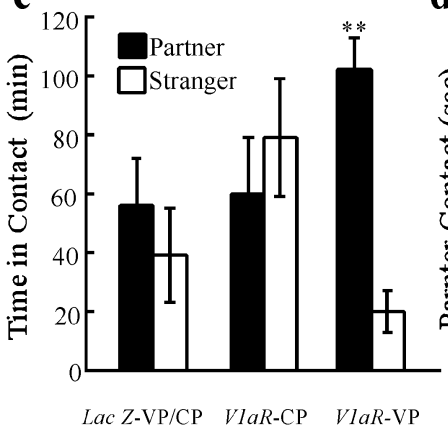

b

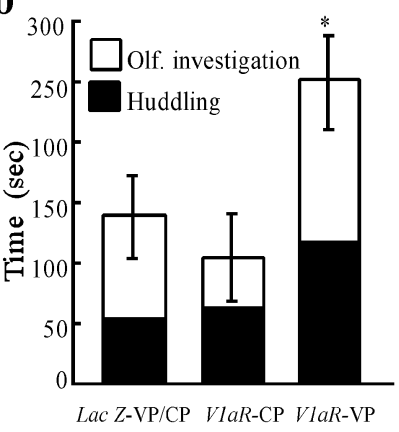

d

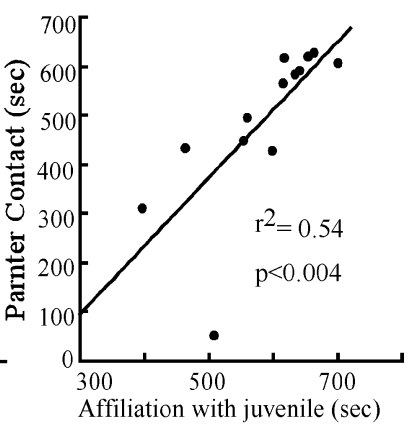

Figure 3. The effects of V1aR gene transfer on anxiety, affiliative behavior, and partner-preference formation in male prairie voles. $a$, Animals with increased V1aR expression in the ventral pallidal area (VlaR-VP) spent significantly less time on the open arms of the elevated plus maze compared with animals injected with the lac $Z$ control virus (lacZ-VP/CP) or the $p v V l a R$ vector into the caudate putamen (VlaR-CP). $b, V 1 a R-V P$ males engaged in more affiliative behavior, defined as either olfactory investigation ( filled bar) or side-by-side contact (open bar), during a 10 min encounter with an unfamiliar juvenile male. $c$, After a $17 \mathrm{hr}$ cohabitation with a nonreceptive female, VlaR-VP males exhibited a partner preference as indicated by spending significantly more time in side-by-side contact with the partner than with the stranger in a $3 \mathrm{hr}$ partnerpreference test. This pattern was not observed in either control group. $d$, Among the VIaR-VP males, there was a significant correlation between the duration of social interactions in the affiliation test and the time spent in side-by-side contact with the partner in the partner-preference test. ${ }^{*} p<0.05 ;{ }^{* *} p<0.005 ;{ }^{* * *} p<0.0001$.

novel female in a simple $3 \mathrm{hr}$ choice test. The sexually naive males were housed with nonestrous females for $17 \mathrm{hr}$ during which mating did not occur. The V1aR-CP and the lacZ-VP/CP males did not consistently display a partner preference, defined as spending twice as much time in the cage with the partner than with the novel female. Of the 17 animals in these groups, 5 exhibited a preference for the partner, 6 exhibited a preference for the stranger, and 6 did not reach the criteria for a preference for either animal. In contrast, 12 of the 13 VlaR-VP males exhibited a partner preference. In addition, as a group, the VlaR-VP animals spent significantly more time in side-by-side contact with their partner than with the stranger (Fig. $3 c)(p<0.005$, Wilcoxon signed rank test). This was not the case for the control groups. In the V1aR-VP group, but not in the control groups, there was a positive correlation between the duration of social interactions in the affiliation test with the juvenile and the time spent in contact with the partner in the partner-preference test (Fig. $3 d)\left(r^{2}=0.54\right.$; $p<0.004)$.

\section{DISCUSSION}

The results from this study also confirm a role for AVP and the $\mathrm{V} 1 \mathrm{aR}$ in the regulation of anxiety, affiliative behavior, and social 
attachment. This report is also the first demonstration that complex social behaviors, such as social attachment, can be facilitated by viral vector gene transfer. In addition, the results demonstrate that animals expressing relatively high levels of $\mathrm{V} 1 \mathrm{aR}$ in the ventral pallidum display higher levels of affiliative behavior and are more likely to form a pair bond than animals with lower levels of receptor in this region. The results are consistent with the hypothesis that species differences in $\mathrm{V} 1 \mathrm{aR}$ expression may explain species differences in social organization, particularly because several monogamous species have higher densities of V1aR in the ventral pallidum than related nonmonogamous species. These data also imply that individual differences in the expression of the VlaR gene could account for individual differences in social behavior and attachment and could have important implications for psychiatric conditions such as autism.

Dense networks of AVP immunoreactive fibers are found in the lateral septum and extend ventrally into the ventral pallidal area of the male prairie vole (Wang et al., 1996). In rats, the septal AVP innervation arises from the bed nucleus of the stria terminalis and the medial amygdala (DeVries and Buijs, 1983). It has been demonstrated recently that AVP infused into the septal area facilitates the formation of partner preferences in the male prairie vole (Liu et al., 2001). Together with the present results, these data suggest that vasopressinergic neurons located in the bed nucleus of the stria terminalis and the medial amygdala regulate partner-preference formation through the release of AVP into the lateral septal and ventral pallidal area, resulting in the activation of ventral pallidal V1aR.

There are two caveats worth noting in our present results. First, $\mathrm{V} 1 \mathrm{aR}$ binding increased in a diffuse region of the medioventral forebrain, sometimes including the portions of diagonal band, ventral lateral septum, and nucleus accumbens. We have focused on the ventral pallidum because this region showed increased binding in all of the experimental animals and because this is the region that expresses the endogenous $V 1 a R$ gene in prairie voles. A second issue is the possibility that regions other than the ventral pallidum are involved in the AVP-dependent regulation of social behavior and pair bonding. V1aRs are also found in the amygdala, thalamus, cingulate cortex, and olfactory bulb. Although our results do not rule out an involvement of these areas, they do demonstrate that increased levels of $\mathrm{V} 1 \mathrm{aR}$ in the ventral pallidum facilitate partner-preference formation.

The V1aR is a G-protein-coupled, seven transmembrane domain receptor. Hepatic V1aR is coupled to $G_{\alpha q 11}$, which activates phospholipase $\mathrm{C}$, thereby increasing inositol triphosphate and intracellular calcium (Wange et al., 1991). Biochemical studies have suggested that the V1aR is also associated with other $\mathrm{G}_{\alpha}$ subunits as well as with phospholipase A (Strakova et al., 1997). Presumably, the behavioral effects of V1aR activation in the ventral pallidal area are the result of the activation of a specific set of second messenger pathways, and are therefore not necessarily specific for AVP or the V1aR. It would be interesting to express in the ventral pallidum other G-protein-coupled receptors that are coupled to the same effector systems in conjunction with the appropriate ligand administration to determine whether the behavioral specificity lies in the $\mathrm{V} 1 \mathrm{aR}$ itself or in the downstream second messenger systems.

Microdialysis studies in rats have demonstrated that vasopressin is released in the brain under certain stressful situations such as after a social defeat or during a forced swim test (Wotjak et al., 1996, 1998). Decreasing V1aR expression using antisense oligonucleotides or infusion of a $\mathrm{V} 1 \mathrm{aR}$ antagonist decreases anxiety in rats, as measured by increased time in the open arms of the elevated plus maze (Landgraf et al., 1995; Liebsch et al., 1996). The present results are consistent with these observations because animals with elevated $\mathrm{V} 1 \mathrm{aR}$ expression in the ventral pallidum exhibited a decrease in time spent in the open arms of the elevated plus maze. These same animals exhibited higher levels of affiliation as measured by increased time investigating and huddling with a juvenile. It is plausible that heightened levels of affiliation are attributable to the increased anxiety. For example, animals that are more anxious may tend to seek the comfort of social contact. However, the lack of a correlation between time spent in the open arms of the plus maze and the duration of affiliative behavior in the VlaR-VP group suggests that the increases in anxiety and affiliation are probably regulated by different mechanisms.

The V1aR-VP males exhibited a strong partner preference after the $17 \mathrm{hr}$ cohabitation without mating. It is important to note that in previous studies from our group, male prairie voles that cohabitated with a female for $24 \mathrm{hr}$ did form partner preferences if mating occurred, but typically did not if mating did not occur (Insel and Hulihan, 1995; Insel et al., 1995). Thus, it seems that by increasing the density of $\mathrm{V} 1 \mathrm{aR}$ in the ventral pallidum, the amount of social stimulation required to form a partner preference was decreased. Also, the probability of forming a pair bond during this short exposure period could have been affected by increased affiliative behavior displayed by the males. Among the VIaR-VP males, there was a correlation between the duration of affiliative behavior with the juvenile and the time spent in contact with the partner. Perhaps the increased social interest expressed in these animals resulted in increased social stimulation, thereby facilitating the formation of the pair bond.

This is the first study to implicate the ventral pallidum in the regulation of social behavior and attachment. It should be noted that the field of $\mathrm{V} 1 \mathrm{aR}$ binding in the prairie vole ventral forebrain is not restricted to the ventral pallidum but likely extends into the ventral lateral septum and substantia innominata. Thus we cannot rule out a role for these structures in the regulation of AVPdependent behaviors. However, functional anatomical studies of the ventral pallidum make it a particularly interesting candidate site for a role in pair-bond formation. The ventral pallidum is a major relay of the shell of the nucleus accumbens and, like the nucleus accumbens, receives dopaminergic input from the ventral tegmental area (Klitenick et al., 1992). This striatopallidal system is an important neurobiological substrate for the rewarding and reinforcing properties of natural stimuli and psychostimulants (McBride et al., 1999). Infusion of psychostimulants directly into the ventral pallidum leads to the development a conditioned place preference for the environment in which the injections were experienced (Gong et al., 1996). Given the abundance of V1aR in the prairie vole ventral pallidum and its role in conditioned place preference, we hypothesize that AVP released during social interactions or mating activates V1aR in the ventral pallidum. Activation of this reward circuitry then reinforces this behavior, leading to an increase in social interactions. In a mating pair, the reinforcement is powerful enough to lead to a conditioned partner preference in the monogamous prairie vole and thereby initiates the formation of a pair bond. The lack of V1aR in the ventral pallidum of nonmonogamous vole species may explain their inability to form partner preferences after mating. There are most certainly other genetic, neurochemical, or anatomical differences between monogamous and nonmonogamous species that contribute to their diverse social behavior; however, the viral vector approach provides an opportunity to test this hypothesis. It should be possible to elevate V1aR expression in the ventral forebrain of nonmonogamous montane or 
meadow voles and determine whether partner preferences are formed.

The role of AVP in facilitating pair bonding in the male prairie vole is remarkably parallel to that of oxytocin in the female prairie vole. In the female it is oxytocin, not vasopressin, that facilitates the formation of the pair bond with the mate (Insel and Hulihan, 1995). Oxytocin antagonist infused into the nucleus accumbens prevents partner-preference formation in the female (Young et al., 2001). In addition, prairie voles have much higher concentrations of oxytocin receptors in the nucleus accumbens than do nonmonogamous vole species (Insel and Shapiro, 1992). Dopamine D2 receptor antagonists infused into the nucleus accumbens also prevent partner-preference formation (Gingrich et al., 2000). Thus it appears that pair bonding is facilitated in a sex-specific manner, by two different neuropeptide systems acting at two separate points in a common neural circuit.

Our results are consistent with the hypothesis that the striatopallidal reward circuitry facilitates certain aspects of affiliation and social attachment, implying common neural pathways for social attachment and the reinforcing effects of drugs of abuse (Panksepp, 1998). A recent functional magnetic resonance imaging study examined the pattern of brain activation and deactivation in human subjects as they viewed photographs of individuals with whom they reported to be romantically in love. The regions of activation were strikingly similar to those activated in studies of cocaine- and $\mu$ opioid agonist-induced euphoria (Bartels and Zeki, 2000). Although the role of vasopressin in human social attachment is unclear and the distribution of $\mathrm{V} 1 \mathrm{aR}$ in the human brain has not been fully described, it is intriguing to consider that plasma vasopressin levels are elevated during sexual arousal in the human male (Murphy et al., 1987). Although increases in plasma AVP released from the posterior pituitary do not necessarily correspond to releases in the brain, these findings raise the possibility that similar neural mechanisms may underlie pairbond formation in rodents and human males.

\section{REFERENCES}

Bartels A, Zeki S (2000) The neural basis for romantic love. NeuroReport 11:3829-3834.

Bartlett JS, Samulski RJ, McCown TJ (1998) Selective and rapid uptake of adeno-associated virus type 2 in brain. Hum Gene Ther 9:1181-1186.

Bester-Meredith JK, Young LJ, Marler CA (1999) Species differences in paternal behavior and aggression in Peromyscus and their associations with vasopressin immunoreactivity and receptors. Horm Behav 36:25-38.

Carter CS (1998) Neuroendocrine perspectives on social attachment and love. Psychoneuroendocrinology 23:779-818.

Carter CS, Getz LL (1993) Monogamy and the prairie vole. Sci Am 268:100-106.

Cho MM, DeVries CC, Williams JR, Carter CS (1999) The effects of oxytocin and vasopressin on partner preferences in male and female prairie voles (Microtus ochrogaster). Behav Neurosci 113:1071-1079.

DeVries GJ, Buijs RM (1983) The origin of vasopressinergic and oxytocinergic innervation of the rat brain with special reference to the lateral septum. Brain Res 273:307-317.

Gingrich B, Liu Y, Cascio W, Wang Z, Insel TR (2000) Dopamine D2 receptors in the nucleus accumbens are important for social attachment in female prairie voles (Microtus ochrogaster). Behav Neurosci 114:173-183.

Gong W, Neill D, Justice JB (1996) Conditioned place preference and locomotor activation produced by injection of psychostimulants in ventral pallidum. Brain Res 707:64-74.

Insel TR, Hulihan T (1995) A gender-specific mechanism for pair bonding: oxytocin and partner preference formation in monogamous voles. Behav Neurosci 109:782-789.

Insel TR, Shapiro LE (1992) Oxytocin receptor distribution reflects social organization in monogamous and polygamous voles. Proc Natl Acad Sci USA 89:5981-5985.

Insel TR, Young LJ (2001) The neurobiology of attachment. Nat Rev Neurosci 2:129-135.

Insel TR, Wang Z, Ferris CF (1994) Patterns of brain vasopressin receptor distribution associated with social organization in microtine rodents. J Neurosci 14:5381-5392.

Insel TR, Preston S, Winslow JT (1995) Mating in the monogamous male: behavioral consequences. Physiol Behav 57:615-627.

Klitenick M, Deutch A, Churchill L, Kalivas P (1992) Topography and functional role of dopaminergic projection from the ventral mesencephallic tegmentum to the ventral pallidum. Neuroscience 50:371-386.

Landgraf R, Gerstberger R, Montkowski A, Probst JC, Wotjak CT, Holboer G, Engelmann M (1995) V1 vasopressin receptor antisense oligonucleotide into septum reduces vasopressin binding, social discrimination abilities, and anxiety-related behavior in rats. J Neurosci 15:4250-4258.

Liebsch G, Wotjak CT, Landgraf R, Engelmann M (1996) Septal vasopressin modulated anxiety-related behaviour in rats. Neurosci Lett 217:101-104.

Liu Y, Curtis JT, Wang ZX (2001) Vasopressin in the lateral septum regulates pair bond formation in male prairie voles (Microtus ochrogaster). Behav Neurosci, in press.

McBride WJ, Murphy JM, Ikemoto S (1999) Localization of brain reinforcement mechanisms: intracranial self-administration and intracranial place-conditioning studies. Behav Brain Res 101:129-152.

Murphy M, Seck1 J, Burton S, Checkley S, Lightman S (1987) Changes in oxytocin and vasopressin secretion during sexual activity in men. J Clin Endocrinol Metab 65:738-741.

Panksepp J (1998) Affective neuroscience (Davidson RJ, Ekman P, Scherer K, eds). New York: Oxford UP.

Peel AL, Zolotukhin S, Schrimsher GW, Muzyczka GW, Reier PJ (1997) Efficient transduction of green fluorescent protein in spinal cord neurons using adeno-associated virus vectors containing cell type-specific promoters. Gene Ther 4:16-24.

Shapiro LE, Dewsbury DA (1990) Differences in affiliative behavior, pair bonding, and vaginal cytology in two species of vole (Microtus ochrogaster and M. montanus). J Comp Psychol 104:268-274.

Strakova Z, Kumar A, Watson J, Soloff MS (1997) A new linear V1a vasopressin antagonist and its use in characterizing receptor/G protein interactions. Mol Pharmacol 51:217-224.

Wang Z, Zhou L, Hulihan TJ, Insel TR (1996) Immunoreactivity of central vasopressin and oxytocin pathways in microtine rodents: a quantitative comparative study. J Comp Neurol 366:726-737.

Wange RL, Smrcka AV, Sternweis PC, Exton JH (1991) Photoaffinity labeling of two rat liver plasma membrane proteins with $\left[{ }^{32} \mathrm{P}\right] \gamma-$ zidoanilido GTP in response to vasopressin: immunologic identification as $\alpha$ subunits of the $\mathrm{Gq}$ class of $\mathrm{G}$ proteins. J Biol Chem 266:11409-11412.

Winslow J, Hastings N, Carter CS, Harbaugh C, Insel T (1993) A role for central vasopressin in pair bonding in monogamous prairie voles. Nature 365:545-548.

Wotjak CT, Kubota M, Liebsch G, Montkowski A, Holsboer F, Newmann I, Landgraf R (1996) Release of vasopressin within the rat paraventricular nucleus in response to emotional stress: a novel mechanism of regulating adrenocorticotropic hormone secretion? J Neurosci 16:7725-7732.

Wotjak CT, Ganster J, Kohl G, Holsboer F, Landgraf R, Engelmann M (1998) Dissociated central and peripheral release of vasopressin, but not oxytocin, in response to repeated swim stress: new insights into secretory capacities of peptidergic neurons. Neuroscience 85:1209-1222.

Wu P, Phillups MI, Bui J, Terwilliger ER (1998) Adeno-associated viral vector-mediated transgene integration into neurons and other nondividing cell targets. J Virol 72:5919-5926.

Young LJ (1999) Oxytocin and vasopressin receptors and species-typical social behaviors. Horm Behav 36:212-221.

Young LJ, Winslow JT, Nilsen R, Insel TR (1997) Species differences in $\mathrm{V} 1 \mathrm{a}$ receptor gene expression in monogamous and non-monogamous voles: behavioral consequences. Behav Neurosci 111:599-605.

Young LJ, Wang Z, Insel TR (1998) Neuroendocrine bases of monogamy. Trends Neurosci 21:71-75.

Young LJ, Nilsen R, Waymire KG, MacGregor GR, Insel TR (1999a) Increased affiliative response to vasopressin in mice expressing the vasopressin receptor from a monogamous vole. Nature 400:766-768.

Young LJ, Toloczko D, Insel TR (1999b) Localization of vasopressin (V1a) receptor binding and mRNA in the rhesus monkey brain. J Neuroendocrinol 11:291.

Young LJ, Lim M, Gingrich B, Insel TR (2001) Cellular mechanisms of social attachment. Horm Behav, in press. 